http://jmscr.igmpublication.org/home/ ISSN (e)-2347-176x ISSN (p) 2455-0450

crossref DOI: https://dx.doi.org/10.18535/jmscr/v8i1.158

\author{
(D) Journal Of Medical Science And Clinical Research \\ IGM Publication \\ An official Publication of IGM Publication
}

\title{
Rare Case of Marchiafava Bignami Disease- A Case Report
}

\author{
Authors \\ Kondeti Ganga Bhavani ${ }^{1^{*}}$, Sarma Y.S ${ }^{2}$, Revathi Potnuru ${ }^{3}$ \\ GSL Medical College, Rajanagaram, Rajahmundry 533296 Andhra Pradesh, India \\ *Corresponding Author \\ Kondeti Ganga Bhavani
}

\begin{abstract}
Marchiafava bignami disease is rare condition characterized by demyelination of corpus callosum. It is seen most often in patients with chronic alcoholism. It is also seen in non alcoholics, for example in patients with malnutrition and poorly controlled diabetes with dramatic fluctuations in serum glucose. If the underlying cause is alcoholism prognosis is poor. It is a rare case of overall prevalence 250 reported cases worldwide. Here we report such a case of 43 year old male presented with seizures and vomiting diagnosed as marchiafava bignami disease. Patient improved on treatment.
\end{abstract}

\section{Introduction}

Marchiafava-Bignami disease is a rare disorder of demyelination or necrosis of the corpus callosum and adjacent subcortical white matter that occurs predominantly in malnourished alcoholics ${ }^{[1]}$.

The course of the disease may be acute, subacute, or chronic and is marked by dementia, spasticity, dysarthria, and inability to walk. Patients may lapse into coma and die, survive for many years in a demented condition, or occasionally recover ${ }^{[2]}$. Lesions appear as hypodense areas in portions of the corpus callosum on computed tomography (CT) and as discrete or confluent areas of decreased $\mathrm{T} 1$ signal and increased $\mathrm{T} 2$ signal on magnetic resonance imaging (MRI) ${ }^{[3] \text {. }}$

Alcohol abusers without liver disease, amnesia, or cognitive dysfunction show thinning of the corpus callosum at autopsy ${ }^{[4]}$ and on $\mathrm{MRI}^{[5,6]}$, suggesting that alcohol or malnutrition damages the corpus callosum commonly in the absence of the necrotic lesions of Marchiafava-Bignami disease. These findings raise the possibility that aggressive nutritional supplementation along with a reduction in drinking can prevent the development of Marchiafava-Bignami disease in alcohol abusers.

\section{Case Report}

A 43 year old male patient presented to the hospital with seizures and vomiting since 3 days after 2 days of binge drinking of alcohol. Patient is known alcoholic and smoker since 20 years. His vitals are blood pressure 200/140mmhg and heart rate of 40beats/min. On central nervous system examination patient is drowsy, arousable to verbal stimuli with normal tone in bilateral upper and lower limbs. Power is 3/5 and deep tendon reflexes are absent in bilateral upper and lower limbs. Bilateral Plantar no response.

On complete blood picture HB: $11 \mathrm{~g} \%$, TC: 7400cumm, platelets: 3.2lakhs/cumm. Renal and liver function tests are within normal limits. ECG showing sinus bradycardia. On $2 \mathrm{~d}$ echo LVEF is 56.6\%. On CXRAY (PA view) no abnormality 
detected and USG abdomen is normal study. MRI brain showed hypointensity involving body and splenium of corpus callosum suggestive of marchiafava bignami disease.

On being diagnosed as marchiafava bignami disease patient was treated with thiamine, B12 supplementation and corticosteroids (injection methyl prednisolone $1 \mathrm{gm}$ intravenously once a day for 3 days) along with anti epileptics and antihypertensives. Patient was improved on treatment.
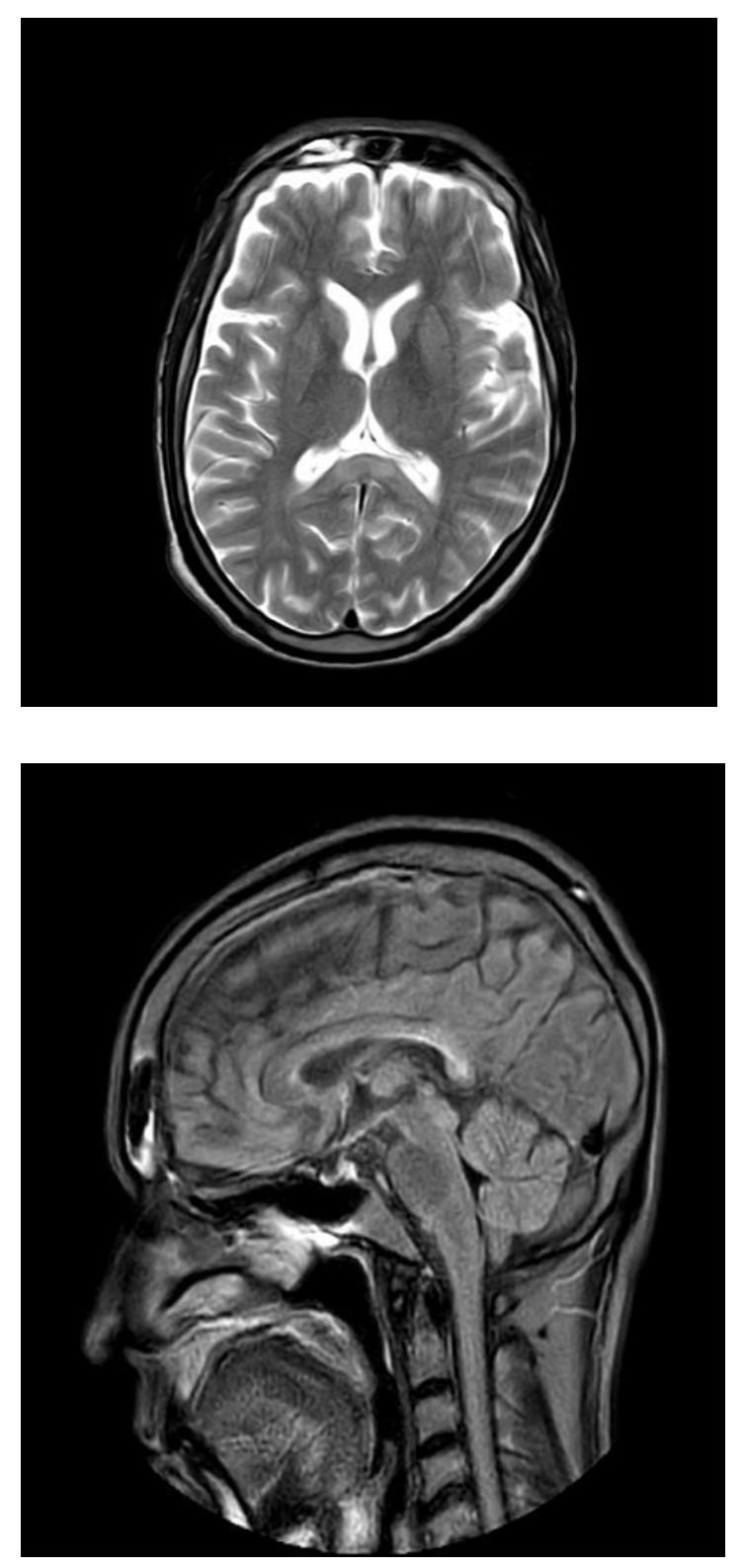

\section{Discussion}

It is a rare condition characterized by demyelination of corpus callosum. It is seen most often in patients with chronic alcoholism.

Two clinical subtypes based on radiological view
Type A: predominant features of coma and stupor with pyramidal tract symptoms, radiologic features involvement of entire corpus callosum.

Type B: normal or mildly impaired mental status with partial or focal collosal lesions.

On MRI: Sand witch sign on T1 imaging is pathognomonic.

On histology: middle portion of myelinated tracts of corpus callosum degenerates.

Treatment considerations include thiamine, corticosteroids and amantadine.

\section{Conclusion}

The present case is diagnosed as marchiafava bignami disease based on history of alcoholism, clinical presentation and MRI finding of hypointensity involving body and splenium of corpus callosum and the patient improved on treatment.

\section{References}

1. Brion, S. Marchiafava-Bignami syndrome. In: Metabolic and Deficiency Diseases of the Nervous System, Part 2, Vinken, PJ, Bruyn, GW (Eds), North-Holland Publishing Company, Amsterdam 1976. p.317.

2. Rosa A, Demiati M, Cartz L, Mizon JP. Marchiafava-Bignami disease, syndrome of interhemispheric disconnection, and righthanded agraphia in a left-hander. Arch Neurol 1991; 48:986.

3. Harper CG, Kril JJ. Corpus callosal thickness in alcoholics. Br J Addict 1988; 83:577.

4. Estruch R, Nicolás JM, Salamero M, et al. Atrophy of the corpus callosum in chronic alcoholism. J Neurol Sci 1997; 146:145.

5. Pfefferbaum A, Lim KO, Desmond JE, Sullivan EV. Thinning of the corpus callosum in older alcoholic men: a magnetic resonance imaging study. Alcohol Clin Exp Res 1996; 20:752. 